\title{
IL LAVORO MANUALE NELLE ESPERIENZE MONASTICHE (EREMITICHE E CENOBITICHE) DEL MEZZOGIORNO RURALE (SECC. VI-XI)
}

\section{PIETRO DALENA}

\author{
UDC: $27-788(450.7) " 05 / 10 "$ \\ 27-523(450.7) )"05/10" \\ Original scientific paper \\ Manuscript received: 04. 11. 2016. \\ Revised manuscript accepted: 07. 02. 2017. \\ DOI: 10.1484/J.HAM.5.113728
}

\author{
P. Dalena \\ Dipartimento di Lingue e Scienze dell'educazione \\ Università della Calabria \\ Via Pietro Bucci \\ 87036 Arcavacata di Rende (Cs), Italy \\ pietro.dalena@unical.it
}

The essay analyzes the function of the manual work in the monastic life between the late antiquity and the Middle Ages in South of Italy. The manual activities, before of the advent of St. Benedict, are little known because of the scarcity of sources, but with the great age of Benedictine monasticism the question of work become of great importance. The first communities strictly follow the rules of the founder, but in the big southern abbeys (as Montecassino and San Vincenzo al Volturno) the main care concerns the management of large land estates. A new impetus to manual labor comes only with the arrival of the Cistercians. Instead, from the tenth century, the work done by the Italian-Greek monks is completely different: they are known for the large land reclamations, for the foundations of monasteries and villages, for the abilities of amanuenses and for the pictorial exercises.

Keywords: Work, Monasticism, The South of Italy, St. Benedict

Generalmente i monachesimi che segnano la storia del Mezzogiorno medievale presentano uno sviluppo coerente con le stagioni politiche e con le peculiarità geografiche di riferimento. Infatti non si può minimizzare l'incidenza degli eventi politici e militari e la natura stessa dei luoghi sulla formazione e sullattività dei monasteri sia latini che d'ispirazione orientale.

Il monachesimo tardoantico del Mezzogiorno d'Italia è scandito da poche esperienze per lo più concentrate in aree urbane o periurbane che esauriscono la loro vitalità già agli inizi del VII secolo. La scarsità di fonti consente solo di delineare un profilo approssimativo del quadro insediativo monastico e del rapporto col territorio, di cogliere qualche aspetto delle fasi di urbanizzazione connesse alle necessità delle comunità e rapsodici spunti del lavoro manuale per razionalizzare le colture ed organizzare l'attività interna al monastero. Una decretale di papa Gelasio (fine V secolo) ai vescovi di Lucania, Calabria e Sicilia, in cui detta alcune disposizioni di carattere generale (sulla promozione dei monaci all'ordine sacro, sul divieto di accogliere schiavi nei monasteri senza l'autorizzazione del loro padrone e sulla scomunica dei conviventi delle vergini consacrate), fa ritenere che sul finire del V secolo il monachesimo o, meglio, alcune forme di vita monastica maschili e femminili vi fossero presenti e, in Calabria, in qualche modo diffuse tanto da far affiorare deviazioni e contrasti ${ }^{1}$.

Al tempo di Gregorio Magno (540 circa-604), una delle fonti principali per il tardoantico, gli insediamenti mo- nastici sparsi in modo disomogeneo e con caratteristiche di difficile diagnosi ricordano la Sicilia e la Campania per opposte ragioni tra le regioni maggiormente interessate al fenomeno $^{2}$. La Calabria, invece, ospitava i due monasteri fondati da Cassiodoro presso Squillace tra il 555 e il 560 (il Castellense sul monte Morcia e il Vivariense alle sue pendici) che divennero centri di spiritualità monastica e culturale, svolgendo un'intensa attività nella formazione dei monaci e, in particolare, nel lavoro manuale della ricopiatura delle opere latine, greche, profane e cristiane ${ }^{3}$. Inoltre è ricordato il monastero di Sant'Arcangelo a Tropea, fatiscente e ricostruito in età bizantina; un altro a Taurianum (Taurianova), che subì in modo destruente l'invasione longobarda tanto da disperdere la comunità monastica, poi in parte raccolta nel monastero di San Teodoro a Messina; e ancora un monastero a Reggio di cui si conosce solo il nome dell'abate, Giovanni, incaricato da papa Gregorio di condurre un'inchiesta tra i monaci dell'isola di Vulcano ${ }^{4}$. Le poche notizie, tratte dalle Epistulae di Gregorio Magno, delineano del primo monachesimo le difficoltà di radicarsi nel territorio e le debolezze morali e organizzative accentuate dalla spinta devastatrice dei nefandissimi longobardi.

Comunque, del monachesimo prebenedettino si conosce ancora molto poco per la nota carenza di fonti; e non sembra che i membri delle primitive comunità monastiche fossero dediti a lavori manuali all'infuori dell'attività amanuense. Così, per esempio, nei monasteri fondati da Martino di Tours «non si esercitava alcun mestiere se non quello di scrivano,

\footnotetext{
${ }^{1}$ G. OTRANTO, Per una storia dell'Italia tardoantica cristiana, Bari, 2009 (Biblioteca tardoantica. Collana del Dipartimento di studi classici e cristiani dell'Università di Bari, 3), p. 433 ss.

${ }^{2}$ Accanto al bel saggio di Carmelina Urso in questo volume si vedano G. VITOLO, Caratteri del monachesimo nel Mezzogiorno altomedievale (secc. VI-IX), Salerno, 1984, p. 6-7; inoltre, la nota di P. DE LEO, Aspetti istituzionali della presenza benedettina in Calabria e Basilicata nell'alto Medioevo, in Montecassino. Dalla prima alla seconda distruzione. Momenti e aspetti di storia cassinese (secc. VI-IX), Atti del II Convegno di studi sul Medioevo meridionale (Cassino-Montecassino, 27-31 maggio 1984), Montecassino, 1987, (Miscellanea Cassinese, 55), p. 441-483; Отranto, Per una storia dell'Italia tardoantica, op. cit. (n. 1), p. 369-373, 433-442.

3 OTRANTO, Per una storia dell'Italia tardoantica, op. cit. (n. 1), p. 433.

${ }^{4}$ Ibidem, p. 435.
} 
a cui venivano adibiti i più giovani; gli anziani si dedicavano all'orazione»". Probabilmente ciò era legato all'estrazione aristocratica di molti personaggi che intraprendevano la vita cenobitica.

Con la Regula Magistri, di poco anteriore a quella benedettina, si hanno le prime attestazioni scritte di lavori manuali nei cenobi occidentali ${ }^{6}$. I monaci potevano svolgere piccoli lavori nel monastero e negli orti, ma da questi non dipendeva il sostentamento della comunità. D’altronde, la preoccupazione principale della Regula Magistri sembra essere la moderazione del lavoro.

\section{IL 'LAVORO’ NELLA CONCEZIONE BENEDETTINA}

Solo con Benedetto da Norcia (per la prima volta in Occidente) il monachesimo latino approccia il lavoro come elemento valoriale sulla via della perfezione. La grande differenza tra la Regola di Benedetto e la Regola del Maestro, che di Benedetto è tra le fonti principali, oltre alla consistenza dei carichi di lavoro (la Regola benedettina rimarca per i monaci la necessità della fatica del lavoro manuale) è nella concezione generale del tempo monastico: nella $R e-$ gula Magistri il tempo è scandito dall'Ufficio divino; nella Regola benedettina il tempo è ripartito tra lavoro e lectio divina, in una concezione che vede il ritmo della vita misurato dall'alternarsi di riposo, preghiera e lavoro manuale. Il lavoro manuale quotidiano è una componente essenziale della visione monastica che Benedetto recupera dalla tradizione basiliana ${ }^{7}$ : se al lavoro si possono dedicare più ore che all'Ufficio divino, e quest'ultimo viene talora condizionato dalle occupazioni manuali ${ }^{8}$.

Nel capo quarantotto della Regola (De opera manuum cotidiana) Benedetto ricorda che in alcune ore del giorno $\mathrm{i}$ monaci debbono dedicarsi al lavoro manuale (in labore manuum), inteso non solo come occupazione nei lavori agricoli, ma anche come attività interna al monastero (cucina, pulizia, infermeria, forno) ed esercizio delle varie arti e professioni che variavano dalle attività amanuensi della compilazione e decorazione dei codici a quelle mediche. E solo in caso di estrema necessità (necessitas loci aut paupertas) i monaci potevano adoperarsi a coltivare la terra e raccoglierne i frutti per realizzare la condizione ideale dello spirito monastico, $\mathrm{i}$ cui principi fondamentali sono contenuti nella formula «vere monachi sunt, si labore manuum suarum vivunt» coerente con l'exemplum dei padri e degli stessi apostoli ${ }^{9}$.

Il lavoro esercitato con umiltà, infatti, per Benedetto acquisisce il carattere di azione sacra, rapportato all'ascesi: è un rimedio all'ozio che è nemico dell'anima: «Otiositas inimica est animae $»^{10}$. Esso non è tanto un mezzo per tenersi occupati, quanto uno strumento indispensabile per realizzare l'ascesi ed elevare l'anima distogliendola dal male. Si tratta di un atto di obbedienza all'abate per il raggiungimento della perfezione ${ }^{11}$. L'attività fisica deve, tuttavia, necessariamente essere bilanciata dall'otium edificante, inteso come tempo da dedicare alla preghiera e alla contemplazione. Benedetto insiste, pertanto, sulla tranquillità che l'animo deve conservare, quindi sulla misura e sulla considerazione delle persone ${ }^{12}$. L'altra indicazione importante è legata al lavoro come azione disinteressata. Soprattutto gli artigiani non devono gloriarsi delle proprie abilità; e il monaco deve essere distaccato dall'opera e dal suo risultato, ché il lavoro non va considerato come mezzo di guadagno e gli oggetti prodotti nelle botteghe monastiche devono essere venduti a buon mercato per favorire gli indigenti ${ }^{13}$. Infine, il monastero deve tendere ad una sorta di autarchia, organizzandosi in modo che al suo interno si trovi tutto l'occorrente alla vita comune: ossia l'acqua, il mulino, l'orto e i vari laboratori, strumenti attraverso cui esercitare il lavoro manuale ${ }^{14}$.

Non è noto quanto la Regola fosse diffusa nel mondo monastico latino, al di fuori della Terra di San Benedetto. E non hanno fondamento le tradizioni locali che vorrebbero alcune comunità fondate da Mauro e Placido (VI secolo), discepoli di Benedetto ${ }^{15}$. Del resto, è stato ben osservato che «è usanza piuttosto diffusa che i monasteri (in particolare quelli più ricchi e potenti) cerchino di costruirsi una nobiltà di origine dimostrando ad ogni costo la loro precoce adozione della Regola, ricevuta addirittura dalle mani stesse di qualche immediato discepolo di san Benedetto» ${ }^{16}$. Ma non si può escludere che la Regola sia stata diffusa occasionalmente, senza una pianificazione. Cosa assai probabile per alcune regioni come la Puglia, dove, l'esperienza benedettina potrebbe essere approdata attraverso Sabino (461-566), vescovo di Canosa, che, ricorda Gregorio Magno, «era solito recarsi dal servo di Dio [Benedetto] ${ }^{17}$.

\footnotetext{
${ }_{5}^{5}$ SULPICIO SEVERO, Vita di Martino, E. Giannarelli, M. Spinelli (a cura di), Torino, 1995, c. X, v. 6, p. 37.

${ }^{6}$ Per il lavoro nella Regula Magistri, si v., in particolare, il cap. 50, in Patrologia latina, 88, col. 1010-1014. Inoltre si rimanda a A. DE VOGÜÉ, La Régle du Maître, Paris, 1964 (Sources chrétiennes, 105-106); ID., Les Régles monastiques anciennes: 400-700, Turnhout, 1986; Regola del Maestro, in G. Turbessi (a cura di), Regole monastiche antiche, traduzione di M. Bozzi, Roma, 1974 (Testi e documenti, 9), p. 367-395.

${ }^{7}$ Patrologia graeca, 31, col. 772, 876, 1009-1018, 1349 .

${ }^{8}$ SAN BENEDETTO, La Regola, A. Lentini (a cura di) (d'ora innanzi: Regola), Montecassino, 1980, c. 48, p. 418-435; inoltre, G. ARCHETTI, "Nihil operi Dei praeponatur". Il tempo dei monaci nel Medioevo, in G. Archetti e A. Baronio (a cura di), "Tempus mundi umbra aevi". Tempo e cultura del tempo tra Medioevo e età moderna, Atti dell'incontro nazionale di studio (Brescia, 29-30 marzo 2007), Brescia, 2008 (Storia, cultura e società, 1), p. 51-80.

${ }_{9}$ Regola, c. 48 , p. 424.

${ }^{10}$ Ibid., c. 48, p. 418-419.

"Ibid., c. 57, p. 502-505.

${ }^{12}$ Ibid., c. 35, p. 324-325; c. 48 , p. $424-425$ e $432-433$.

${ }^{13}$ Ibid., c. 57, p. 502-507.

${ }^{14}$ Ibid., c. 69, p. 610-611.

${ }_{15}$ È noto quanto la fantasia agiografica di Pietro, diacono di Montecassino, abbia prodotto fioretti edificanti sulla presenza di Placido nelle regioni meridionali. Per quanto riguarda la Calabria, Placido viene confuso con l'omonimo martire siciliano ricordato nel martirologio Geronimiano (Bibliotheca Sanctorum, vol. X, sub voce); inoltre sulla presunta attività missionaria di Placido cfr. Die Chronik von Montecassino, H. Hoffmann (ed.), in Monumenta Germaniae Historica, Scriptores, XXXIV, Hannover, 1980, p. 18-19; e sull'attività di falsario di Pietro Diacono si rimanda a E. CASPAR, Petrus Diaconus und die Monte Cassiner Fälschungen, Berlino, 1909, p. 47-72.

${ }^{16}$ G. PENCO, La prima diffusione della Regola di S. Benedetto, Roma 1957 (Studi Anselmiana, 42), p. 321-345.

${ }^{17}$ «Praeterea antistes Canusinae ecclesiae ad eundem Domini famulum venire consueverat, quem vir Dei pro vitae suae merito valde diligebat» (GRÉGOIRE LE GRAND, Dialogues, II, lib. II, A. de Vogüé (ed.), Texte critique et notes par P. Antin, Paris, 1979 (Sources chrétiennes, 260), p. 184-185.
} 
Ma se l'ideale di vita monastica benedettina cominciava ad essere conosciuto e, forse, anche a diffondersi, il dettato della Regola veniva generalmente disatteso, persino nella Terra di san Benedetto. Nelle grandi abbazie benedettine del Mezzogiorno longobardo altomedievale, San Vincenzo al Volturno e Montecassino, i monaci sembrano essere più interessati alla gestione degli enormi patrimoni fondiari dispersi su gran parte del territorio del principato di Benevento che al lavoro manuale ${ }^{18}$. Almeno questo fa pensare la documentazione disponibile. Di grande interesse, per esempio, è il resoconto delle produzioni fatto dal monaco Sabatino di San Vincenzo al Volturno, cellario della curtis di Capriati (poco distante da Isernia). All'indomani della distruzione del cenobio, il cellario vulturnense ricorda come da quei tenimenti monastici, di cui era stato preposito (prepositus) per molto tempo, ogni anno trasferiva al cenobio di San Vincenzo al Volturno centum tritici modia et quadraginta porcos $^{19}$. La cella benedettina si configurava come una vera e propria azienda agricola con accentuato sviluppo nel settore dell'allevamento suino e nella coltivazione delle terre, dove, sotto la vigilanza del preposito, erano impiegati contadini dipendenti e servi.

Montecassino sin dall'alto Medioevo deteneva un patrimonio vastissimo, con possedimenti diffusi in tutto il Mezzogiorno longobardo, che si ampliò notevolmente dopo il 774 con la donazione del ricco monastero beneventano di Santa Sofia effettuata dal principe di Benevento Arechi II ${ }^{20}$. A Santa Sofia faceva capo una rete di possedimenti (notevolmente estesa tra Campania, Sannio, Puglia e Basilicata) declinata in rilevanti latifondi disposti lungo direttrici viarie ben collegate a Benevento, che, principale ganglio viario del Mezzogiorno, si configurava come terminale delle produzioni provenienti dai centri curtensi ${ }^{21}$. La presenza di praepositi cassinesi presso il monastero beneventano, tra i quali si ricorda Bassacio (833-835) che fu poi abate di Montecassino dall'838 all'856, è indice di un controllo ben saldo da parte dell'archicenobio sulla fondazione arechiana.

Del resto, proprio il forte legame con i longobardi non consentì che la riforma monastica (tesa a reprimere abusi troppo stridenti), vagheggiata da Carlo Magno e attuata da Ludovico il Pio con i decreti del sinodo di Aquisgrana (817), vi attecchisse e ne orientasse la vita monastica, anche per ciò che riguarda il lavoro manuale ${ }^{22}$. Circa tre secoli dopo la fondazione di Montecassino, la feconda azione riformatrice di Benedetto d'Aniane (750 ca.-821) che influenzò i sovrani carolingi, confluita nel Capitulare monasticum (817), ebbe come conseguenza la ricomposizione dell'unità spirituale e culturale dell'Europa carolingia. La sua concezione del lavoro manuale (tra gli aspetti nevralgici della riforma della Regola benedettina che condensava tutta la tradizione monastica, con particolare richiamo agli insegnamenti di Basilio di Cesarea) si innervava di esperienza gratificante della quotidianità monastica e di ascesi spirituale esercitate sia all'interno del monastero, sia nelle attività campestri che ne accrescevano la base economica e il loro potere politico. Tuttavia se Benedetto d'Aniane fu protagonista nell'orientare l'espansione del monachesimo benedettino in Europa, la sua riforma non attecchì nel Mezzogiorno d'Italia che non registra effetti, neanche indiretti. Del resto, la sua missione rinnovatrice non riguardava il Mezzogiorno, dove Montecassino agli inizi del IX secolo godeva di forte carisma spirituale ed esercitava una non trascurabile influenza politica ${ }^{23}$.

Dal IX secolo nel Mezzogiorno sono sempre più frequenti le notizie sull'esistenza di monasteri latini della cui organizzazione si sa ben poco. Essi di solito nascono in modo spontaneo, vivono e si sviluppano con fisionomia chiaramente pluralistica, con un proprio codice di comportamento diverso da quello di altre comunità similari, ma non tutti si configurano come "benedettini", nel senso che non adottano le norme benedettine espresse dalla Regola ${ }^{24}$. A volte, invece, sostituiscono fondazioni di rito italo-greco. Per esempio, tra le dipendenze cassinesi del IX secolo, è ricordato il monastero femminile di Santa Maria di Cosenza, in origine di rito bizantino, che sarebbe passato a Montecassino tra l'858 e l'867 quando ne fu ordinata la ricostruzione, come dimostrerebbe un documento apocrifo del 18 aprile dell' $863^{25}$. Dotato di un cospicuo patrimonio formato da curtis e casalibus in cui vivevano servi e ancillae, secondo il modello della 'cella benedettina', formava una vera e propria azienda agricola in cui i dipendenti laici curavano l'allevamento e coltivavano le terre monastiche ${ }^{26}$.

Gestori di grandi patrimoni, per lo più provenienti dai ranghi dell'aristocrazia longobarda e franca, i monaci di San Benedetto e di San Vincenzo al Volturno dovevano praticare ben poco il lavoro dei campi. E il lavoro manuale sarebbe stato limitato agli ortora monastici e, forse, ad alcune opere di artigianato. Infatti, gli scavi condotti presso l'importante abbazia vulturnense hanno riportato alla luce testimonianze

\footnotetext{
${ }^{18}$ Per la consistenza dei beni patrimoniali del cenobio cassinese e l'estensione della sua giurisdizione si rimanda a P. DALENA, La "Terra Sancti Benedicti" tra Europa e Mediterraneo, in P. Dalena e C. Urso (a cura di), "Ut sementem feceris, ita metes". Studi in onore di Biagio Saitta, Acireale-Roma, 2016 (Analecta humanitatis, 31), p. 259-274.

${ }_{19}$ Chronicon Vulturnense del monaco Giovanni scritto intorno all'anno 1130, M. Oldoni (a cura di), I, Cerro al Volturno (Is), 2011, p. 372-373, a. 881.

${ }^{20}$ Chronicon Sanctae Sophiae, J.M. Martin (a cura di), Roma, 2000 (Fonti per la storia d'Italia, Rerum Italicarum Scriptores, 3), p. 49-50.

${ }^{21}$ Per la strutturazione del patrimonio di Santa Sofia si rimanda ad A. DI MURO, Economia e mercato nel Mezzogiorno longobardo (secc. VIII-IX), Salerno, 2009, p. 50-56.

${ }^{22}$ Più in generale si rimanda alle considerazioni di G. PENCO, Storia del monachesimo in Italia. Dalle origini alla fine del Medioevo, Milano, 1983 (Complementi alla storia della Chiesa diretta da H. Jedin), p. 160 ss; inoltre, v. P. RICHÉ, Il cristianesimo nell'Occidente carolingio dalla metà del secolo VIII alla fine del secolo IX, in G. Dagron, P. Riché e A. Vauchez (in a cura), Vescovi, monaci e imperatori (610-1054), ed. it. G. Cracco (a cura di), Roma, 1999 (Storia del cristianesimo. Religione, politica, cultura, 4), p. 690-766.

${ }^{23}$ PH. SCHMITZ, L'influence de S. Benôit d'Aniane dans l'histoire de l'ordre de S. Benôit, in Il monachesimo nell'alto Medioevo e la formazione della civiltà occidentale, IV Settimana del Centro italiano di studi sull'alto medioevo (Spoleto, 8-14 aprile 1956), Spoleto, 1957, p. 401-415.

${ }^{24}$ G. LUNARDI, L'ideale monastico e l'organizzazione interna dei monasteri, in C.D. Fonseca (a cura di), L'esperienza monastica benedettina e la Puglia, Atti del convegno di studio organizzato in occasione del XV centenario della nascita di San Benedetto (Bari-Noci-Lecce-Picciano, 6-10 ottobre 1980), I, Galatina, 1983, p. 140, 142.

${ }^{25}$ F. RUSSO, Regesto Vaticano per la Calabria, I, Roma, 1974, nn. 92 e 94.

${ }^{26}$ Ibidem.
} 
di attività artigianali molto raffinate nei pressi del cantiere abbaziale, dove non è improbabile che qualche monaco vi fosse addetto ${ }^{27}$. Di certo l'attività lavorativa dei monaci di Montecassino e di San Vincenzo nell'alto Medioevo era legata prevalentemente agli scriptoria, dove si producevano eleganti codici, anche miniati.

Ma per realizzare l'ideale monastico, incentrato sulla fuga dal mondo, il tempo maggiore era comunque dedicato alla preghiera, che, nell'aspettativa dei fedeli, era il primo "servizio sociale” per guadagnarsi il viatico per il cielo. Pertanto i monaci non solo pregavano «pro imperiale magnitudine $»^{28}$, ma anche «Deum nobis implorant propitium» $»^{29} \mathrm{e}$ «pro nostra omniumque fidelium salute quotidie militant Creatori» ${ }^{30}$.

La diffusione più omogenea del monachesimo benedettino nel Mezzogiorno si verifica a partire dalla seconda metà dell'XI secolo, con forme di aggregazione e di spiritualità del tutto nuove rispetto al monachesimo tardoantico ${ }^{31}$. Caratteristiche che si richiamano non necessariamente alla riforma cluniacense, che pure approdò a Cava già verso la fine dell'XI secolo, ma sviluppano autonome forme insediative che sperimentano anche rapsodiche forme di attività manuale ed intellettuale, quest'ultima intesa sia come preghiera che come esercizio della pittura e della miniatura.

D'altra parte, pur riconoscendo ai cluniacensi una funzione attiva e partecipativa, si è accentuata l'attenzione sul ruolo svolto da Montecassino e San Vincenzo al Volturno, che, sin dal IX secolo, introdussero negli schemi istituzionali il modello dell'abbazia esente dalla giurisdizione episcopale e alimentarono una serie di filiazioni che spesso agivano in modo centrifugo e del tutto autonome accentuandone la connotazione rurale. Ma sino alla metà dell'XI secolo gli effetti della Regola benedettina (tranne nelle aree di tradizione carolingia permeate dall'azione politica e riformatrice di Benedetto di Aniane e nella Terra Sancti Benedicti) sono ancora poco o nulla evidenti, se si escludono i due grandi monasteri del Mezzogiorno longobardo appena ricordati.

Diverso, invece, l'atteggiamento del monachesimo latino nel Mezzogiorno dalla metà dell'XI secolo, quando, con l'auspicio normanno, comprime l'esperienza monastica italo-greca riducendone poco a poco gli spazi vitali. Esso si organizza in forme generalmente cenobitiche, pur cedendo in alcuni casi alle suggestioni eremitiche del monachesimo italo-greco (bizantino) ben radicato nel territorio. Infatti, forme di vita cenobitica ed eremitica dei monachesimi coesistono in alcune regioni. Soprattutto nelle regioni dove le peculiarità morfologiche favorirono maggiormente l'esperienza monastica italo-greca (Calabria, Basilicata, Puglia e Sicilia) si condividevano pratiche eremitiche, che, nell'esercizio della vita solitaria, sublimata dalla preghiera e dal lavoro manuale, realizzavano attraverso l'hesuchía lo stato di perfezione monastica: l'ascesi. Ne è riprova sul finire dell'XI secolo la lettera di Bruno di Colonia a Raoul il Verde, prevosto del Capitolo di Reims, invitato a raggiungerlo in Calabria, nella zona delle Serre, per condividere nella preghiera l'amenità dei luoghi solitari ${ }^{32}$.

Ma se si eccettuano poche esperienze eremitiche, tra cui quelle di Guglielmo da Vercelli e di Giovanni Scalcione da Matera (fondatori della Congregazione benedettina di Montevergine e della Congregazione degli eremiti Pulsanesi con caratteristiche cenobitiche), maturate tra la fineXI e la prima metà del XII secolo anche in contesti rupestri grecizzati della Puglia con accentuata tendenza eremitica e pauperistica che rese il lavoro agricolo obbligatorio a tutti i monaci33, il Mezzogiorno verso la fine dell'XI secolo, su impulso delle potenti abbazie cassinese e cavense, comincia a latinizzarsi organizzandosi in formazioni di tipo cenobitico, variabili da luogo a luogo. E se Montecassino puntava perspicuamente sul monopolio delle attività assistenziali e intellettuali, Cava, invece, apprezzava maggiormente la direzione delle attività manuali (dalle attività agropastorali a quelle amanuensi) che consentivano un deciso ampliamento della giurisdizione e un cospicuo sviluppo economico.

Le filiazioni cavensi e cassinesi del Meridione, funzione del progetto di latinizzazione della Sede apostolica, avviarono una radicale trasformazione del paesaggio agrario attraverso i villani ascrittizi. Sono frequenti le donazioni di chiese monastiche italo-greche alle comunità latine cui venivano legati villani con la formula/clausola «et quot homines adducere et ponere in terra praedictorum monasteriorum ad habitandum volueritis (...) et servitium faciant»34. I rustici prestavano il loro servitium anche nelle peschiere monastiche; e muratori laici costruivano chiese sotto la supervisione monastica ${ }^{35}$. Ai religiosi spettava il compito di affrescare le pareti delle chiese sub divo e rupestri di cui le epigrafi dedicatorie ne ricordano la committenza e l'operatore (fig. 5). Ma nelle chiese rupestri anche in età normanna è ricordata ancora prevalente la committenza monastica greca o di origine greca degli affreschi o del restauro, come il monaco Nilo autore di un affresco nella chiesa dei SS. Eremiti a Palagianello o il monaco Paolo, committente, rappresentato in ginocchio ai piedi del Cristo nella chiesa di San Giorgio a Grottaglie ${ }^{36}$.

\footnotetext{
${ }^{27} \mathrm{Si}$ veda ad esempio R. HODGES, Light in the Dark Ages. The Rise and Fall of San Vincenzo al Volturno, London, 1997.

${ }^{28}$ F. TRINCHERA, Syllabus graecarum membranarum, Napoli, 1865, p. 24-25, doc. n. XXIII.

${ }^{29}$ P. DE LEO, Le carte del monastero dei Santi Niccolò e Cataldo in Lecce (secc. XI-XVII), Lecce, 1978, p. 18, doc. 5.

$3^{30} \mathrm{~F}$. UGHELLI, Italia Sacra sive de episcopis Italiae et insularum adiacentium, VII, Venetiis, 1721, col. 1130D.

${ }^{31}$ DALENA, La "Terra Sancti Benedicti", op. cit. (n. 18), p. 259-274.

$3^{2}$ P. DALENA, Calabria Medievale. Ambiente e istituzioni (secoli XI-XV), Bari, 2015, p. 185.

33 F. PANARELLI, Dal Gargano alla Toscana. Il monachesimo riformato latino dei Pulsanesi (secoli XII-XIV), Roma, 1997 (Nuovi studi storici, 38 ), p. 44 ss. ${ }^{34}$ Per esempio, nel maggio 1081 Riccardo Senescalco dona al monastero della SS. Trinità di Cava alcuni monasteri italo-greci dismessi (Sant'Angelo e San Vito) in territorio di Mottola e la chiesa di Santa Lucia in territorio di Massafra. E per servizio lega al monastero di Sant'Angelo tre villani di Mottola e alla chiesa di Santa Lucia due villani di Massafra [P. DALENA, Da Matera a Casalrotto. Civiltà delle grotte e popolamento rupestre (secc. X-XV), Galatina, 1990, p. 151-152, doc. 1; inoltre C. Carlone, L. Morinelli, G. Vitolo (a cura di), Codex diplomaticus Cavensis, XI, 1081-1085, Badia di Cava, 2015, p. 25-27, doc. 9].

35 DALENA, Da Matera a Casalrotto, op. cit. (n. 34).

${ }^{36}$ A. GUILLOU, Aspetti della civiltà bizantina in Italia, Bari, 1976, p. 372-373; inoltre, P. DALENA, Culto dei santi nelle aree rupestri e tramiti viari, in E. Menestò (a cura di), Agiografia e iconografia nelle aree della civiltà rupestre, Atti del V Convegno internazionale sulla civiltà rupestre (Savelletri di Fasano (Br), 17-19 novembre 2011), Spoleto, 2013, p. 63-78.
} 


\section{IL MONACHESIMO BIZANTINO O ITALO- GRECO}

Un maggiore e più fecondo impulso alla monacazione (sperimentata nelle tre forme ricordate nella Scala Paradisii di Giovanni Climaco, vissuto tra il 575 e il 650 circa) si ebbe in alcune regioni dell'Italia meridionale tra il X e la prima metà dell'XI secolo, in particolare in quella calabro-lucana, in Puglia e in Sicilia, dove monaci d'ispirazione orientale praticando l'anacoresi anche in forme cenobitiche col proprio lavoro manuale fondavano monasteri, curavano l'accoglienza e l'attività scrittoria, urbanizzavano il paesaggio e vi sviluppavano una fertile economia agro-pastorale. Sono abbastanza diffusi gli asceteri rupestri abitati da anacoreti (soprattutto in Calabria) e le lavre di piccole comunità monastiche italo-greche dedite al dissodamento e a varie attività

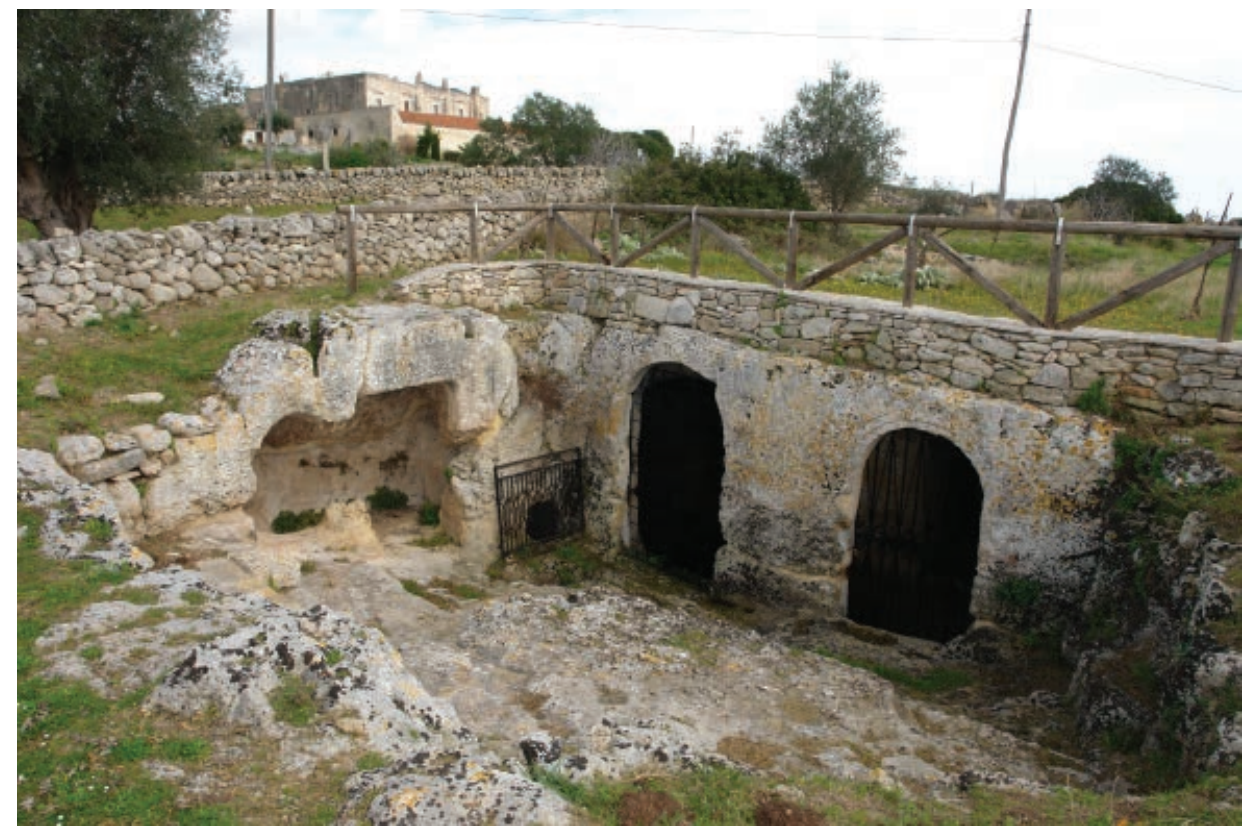

Fig. 1. Mottola (TA): Chiesa monastica italo-greca di Sant'Angelo di Casalrotto, ingresso e pronao. campestri basate sul lavoro manuale edificante ${ }^{37}$.

I monasteri divennero referenti religiosi ed economici dei villaggi (anche rupestri) che si organizzavano attorno e si alimentavano di esperienze e cultura monastica anche in tempi di difficoltà. Il lavoro manuale (la cui importanza è rimarcata tanto nelle diataxeis di Basilio Magno di Cesarea, quanto nei Typikà dei monasteri italo-greci dell'Italia meridionale), infatti, era finalizzato al bene comune della comunità monastica e del villaggio nella sua duplice funzione di laboratorio economico e di esercizio spirituale espresso tramite la pittura, la miniatura e, in genere, l'attività amanuense (figg. 1-4).

Per Basilio l'ideale del monaco era di scoprire la misura tra Bíos practicós e Bíos teoretikós, cioè tra la vita attiva e la vita contemplativa, per raggiungere Dio e assicurarsi la salvezza dell'anima ${ }^{38}$. Il padre del monachesimo bizantino nella sua diataxeis accentua la necessità del lavoro manuale capace di distrarre dalle difficoltà morali e dalle tentazioni quotidiane. Infatti è necessario che «il monaco si dedichi a lavori appropriati al suo stato, di quelli che non comportano alcun traffico o troppo grandi fastidi o guadagni scandalosi, di quelli che possono essere eseguiti anche all'interno del monastero, dove ci troviamo più spesso, affinché, da una parte, il lavoro sia compiuto e che, d'altra parte l'esuchia sia conservata» 39 .

Pertanto, sono i lavori dei campi quelli che si adattano meglio allo stato monacale. Gli stessi precetti si trovano in Doroteo di Gaza (VI secolo) e in Teodoro Studita, riformatore del monachesimo (IX secolo). In Italia meridionale (dalla Sicilia alla Puglia e alla Campania meridionale) sino al $\mathrm{X}$ secolo, e prima della crisi politica che nella seconda metà dell'XI secolo segnò la fine della dominazione bizantina, i monaci italo-greci dissodavano e disboscavano direttamente, trasformando boschi e lande in coltivi. Saba e Macario

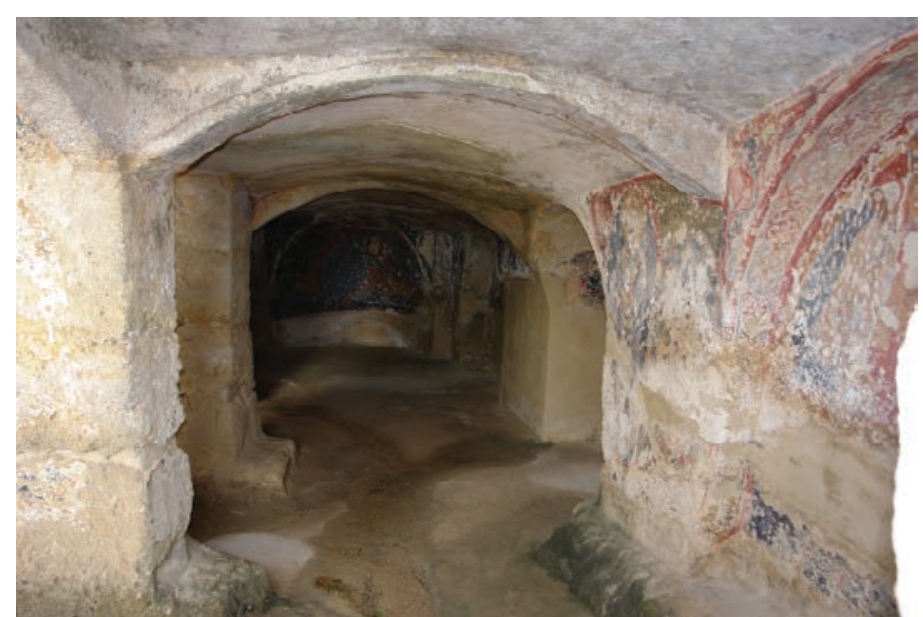

Fig. 2. Mottola (TA): Chiesa monastica italo-greca di Sant'Angelo di Casalrotto, interno.

si insediarono nell'inospitale Mercurion e vi costruirono un monastero dopo aver abbattuto alberi, bruciato sterpaglie, risanato la terra che guadagnarono alla coltivazione e alla produttività ${ }^{\circ}$.

Generalmente i monaci d'ispirazione bizantina nel Meridione d'Italia si distinguono per l'attività di dissodamento che consente di trasformare terreni incolti o dominati dalla boscaglia in coltivi e di renderli adatti ad ospitare un monastero. Saba e Macario giunti nella regione selvosa del Mercurio, in Calabria, notavano che i monaci/asceti abitavano «in montibus cavernisque». E su segnalazione del preposito Niceforo si insediarono in un luogo selvoso «silvestris plantis occupatum» chiamato Ctisma, dove era un santuario dedicato a San Michele Arcangelo, che liberarono dalla boscaglia e ricostruirono a fundamentis ${ }^{41}$ (fig. 6-8). Poi, spintisi in Lucania nella regione del Latinianon, lungo

\footnotetext{
${ }_{37}$ Cfr. bibliografia in P. Dalena (a cura di), Medioevo rupestre. Strutture insediative nella Calabria settentrionale, Bari, 2007, p. $245-260$.

${ }^{38}$ Medioevo rupestre, op. cit. (n. 37), p. 270 ss.

39 A. GUILLOU, Il monachesimo greco in Italia meridionale e in Sicilia nel Medioevo, in L'eremitismo in Occidente nei secoli XI e XII, Atti della seconda Settimana internazionale di studio (Mendola, 30 agosto-6 settembre 1962), Milano, 1965 (Miscellanea del Centro di Studi Medievali, IV), p. 361.

${ }^{40}$ GUILLOU, Aspetti della civiltà bizantina, op. cit. (n. 36), p. 273.

${ }^{41}$ Historia et laudes SS. Sabae et Macarii iuniorum e Sicilia auctore Oreste Patriarca Hierosolymitano, I. Cozza Luzi (ed.), Roma, 1893, p. 8, § III.
} 


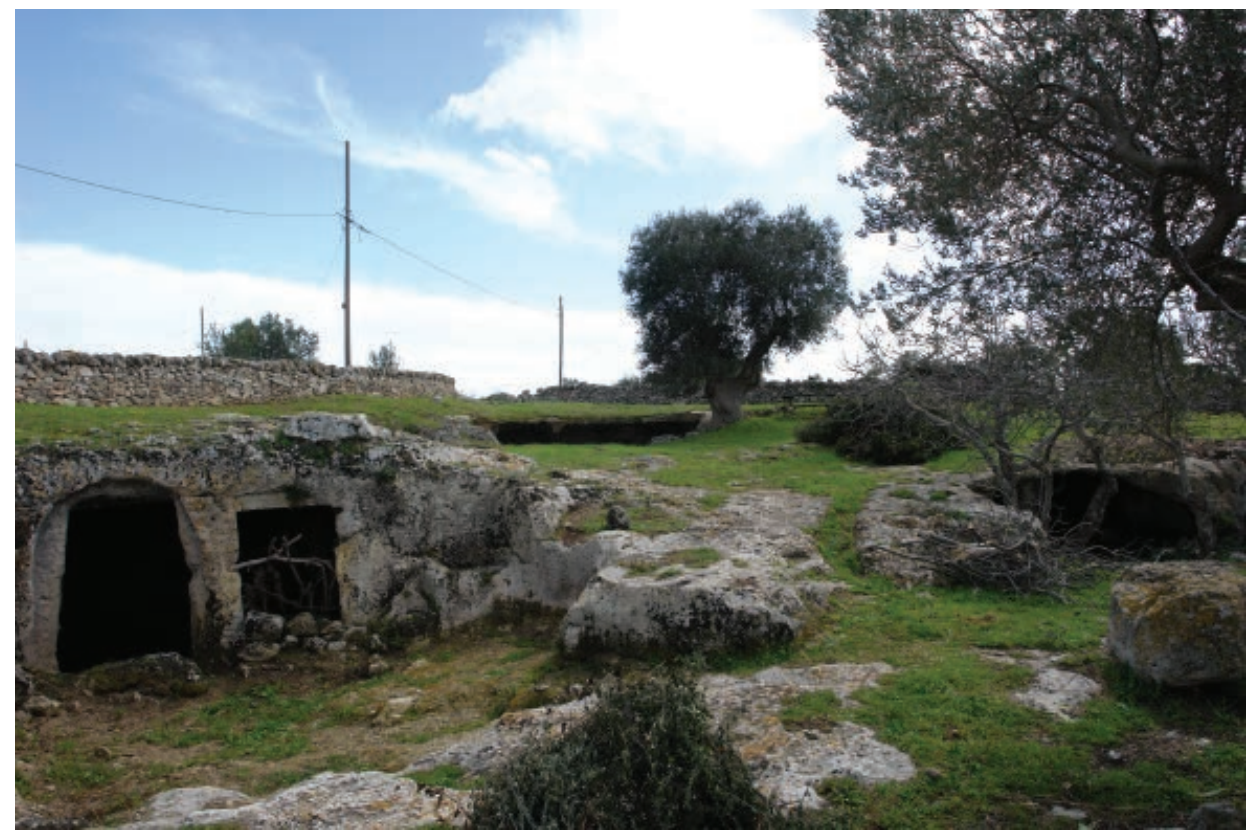

Fig. 3. Mottola (TA): Casalrotto. Monastero e villaggio rupestri materialmente e spiritualmente. E per questo André Guillou, con grande efficacia, ha sintetizzato, che esso ne è stato il lievito, prima di divenire il reliquario delle tradizioni bizantine ${ }^{43}$.

L'attività manuale dei monaci italo-greci nell'Italia meridionale si rivelò decisiva nell'avviare il processo di urbanizzazione e di riqualificazione del territorio che consentì alle comunità rurali di organizzarsi in comuni fiscali (coria) e ridurre i rischi delle guerre permanenti e le difficoltà del paesaggio aspro. Infatti, sebbene frenati dalle scorrerie dei saraceni che riuscirono persino ad insediarsi nel cuore della Lucania, a Pietrapertosa (sud-ovest di Tricarico), e ad impadronirsi delle terre del territorio di Tricarico ${ }^{44}$, nella seconda metà del X secolo i monaci greci dissodatori, aprendo nuovi spazi, furono protagonisti dell'espansione antropica e della crescita demografica,

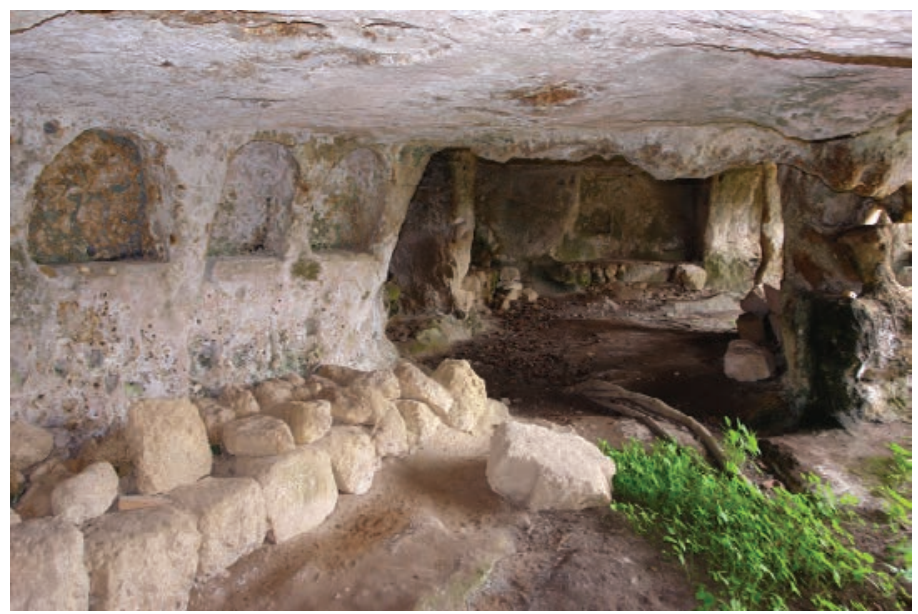

Fig. 4. Mottola (TA): Casalrotto. Monastero rupestre italo-greco.

il fiume Sinni, individuarono un luogo adatto per un insediamento monastico per la presenza di abbondanti risorse idriche e, in particolare, di un'edicola dedicata al beato Lorenzo. Col lavoro manuale (suis manibus) tagliarono alberi e arbusti, bruciarono le sterpaglie e bonificarono il terreno per renderlo ospitale e adatto alla coltivazione ${ }^{42}$. Il lavoro servì a far crescere queste fondazioni, di cui alcune (Carbone, San Giovanni Terista a Bivongi, San Nicola di Casole) divennero importanti centri di potere economico feudale. Il monachesimo italo-greco si affermava come fenomeno rurale per la condivisione del lavoro materiale e per la vicinanza alle popolazioni contadine che da esso dipendevano favorirono la formazione di nuovi villaggi, costruirono chiese e piccoli monasteri, rupestri o subdiali, sottrassero terreno coltivabile al dominio della foresta e dell'incolto, realizzarono un funzionale reticolo viario di servizio.

È significativa la testimonianza del monaco Jonas, che, verso la metà del X secolo, dissodò alcune terre nel territorio di Tricarico donandole, poi, nel 983 al monastero della Teotokos del Rifugio: l'igumeno vi trasferì dei contadini esenti da obblighi fiscali (eleuteri) che urbanizzarono il territorio e organizzarono il villaggio poi iscritto come comune fiscale (corion) nei registri dell'amministrazione bizantina ${ }^{45}$. Una vicenda analoga, anche se più complessa, riguarda il monastero di San Nicola di Cyr-Zosimo che si trasformò in villaggio e poi in paese ${ }^{46}$. Monaci e asceti, per sfuggire alle incursioni saracene, dalla Calabria meridionale e dalla regione montuosa del Mercurio si spinsero nelle valli dell'Agri e del Sinni, dove «deserta quaerebant loca, ad monasteria catervatim confluentes ut saltem panis sibi ad cibum porrigeretur ${ }^{47}$. In quelle valli divennero referenti religiosi e culturali delle popolazioni locali e attivi operatori agricoli che trasformarono il paesaggio e resero le terre produttive. Quasi tutti i monaci provenienti dalla Sicilia, in particolare dal monastero di San Filippo di Agira, attraverso la Calabria portarono in Lucania parenti o compagni con cui avviarono la prima fase di antropizzazione: san Saba, seguito dall'intera famiglia, dopo aver liberato un luogo silvestre, fondò un monastero sulle rive del Sinni, nei pressi di un kastéllion «valde munitum», che fortificò per timore dei saraceni e delle bande di malfattori ${ }^{4}$; Vitale di Castronovo ebbe come

\footnotetext{
${ }^{42}$ Ibidem, p. 17-18, § IX.

${ }_{43}$ GUILLOU, Aspetti della civiltà bizantina, op. cit. (n. 36), p. 292. 1975, p. 40. Taranto, 1977, p. 34.

${ }^{46}$ TRINCHERA, Syllabus graecarum membranarum, op. cit. (n. 28), p. 45-47, n. XXXVII.

${ }^{47}$ Historia et laudes SS. Sabae et Macarii iuniorum, op. cit. (n. 41), p. 25.

${ }^{8}$ Ibidem, p. 17-18.
}

${ }^{44}$ A. GUILLOU, La seconda colonizzazione bizantina nell'Italia meridionale. Le strutture sociali, in C. D. Fonseca (a cura di), La civiltà rupestre medioevale nel Mezzogiorno d'Italia. Ricerche e problemi, Atti del primo convegno internazionale di studi (Mottola-Casalrotto, 29 settembre-3 ottobre 1971), Genova,

${ }^{45}$ A. GUILLOU, Longobardi, Bizantini e Normanni nell'Italia meridionale: continuità o frattura?, in C.D. Fonseca (a cura di), Il passaggio dal dominio bizantino allo Stato normanno nell'Italia meridionale, Atti del secondo convegno internazionale di studi (Taranto-Mottola, 31 ottobre-4 novembre 1973), 


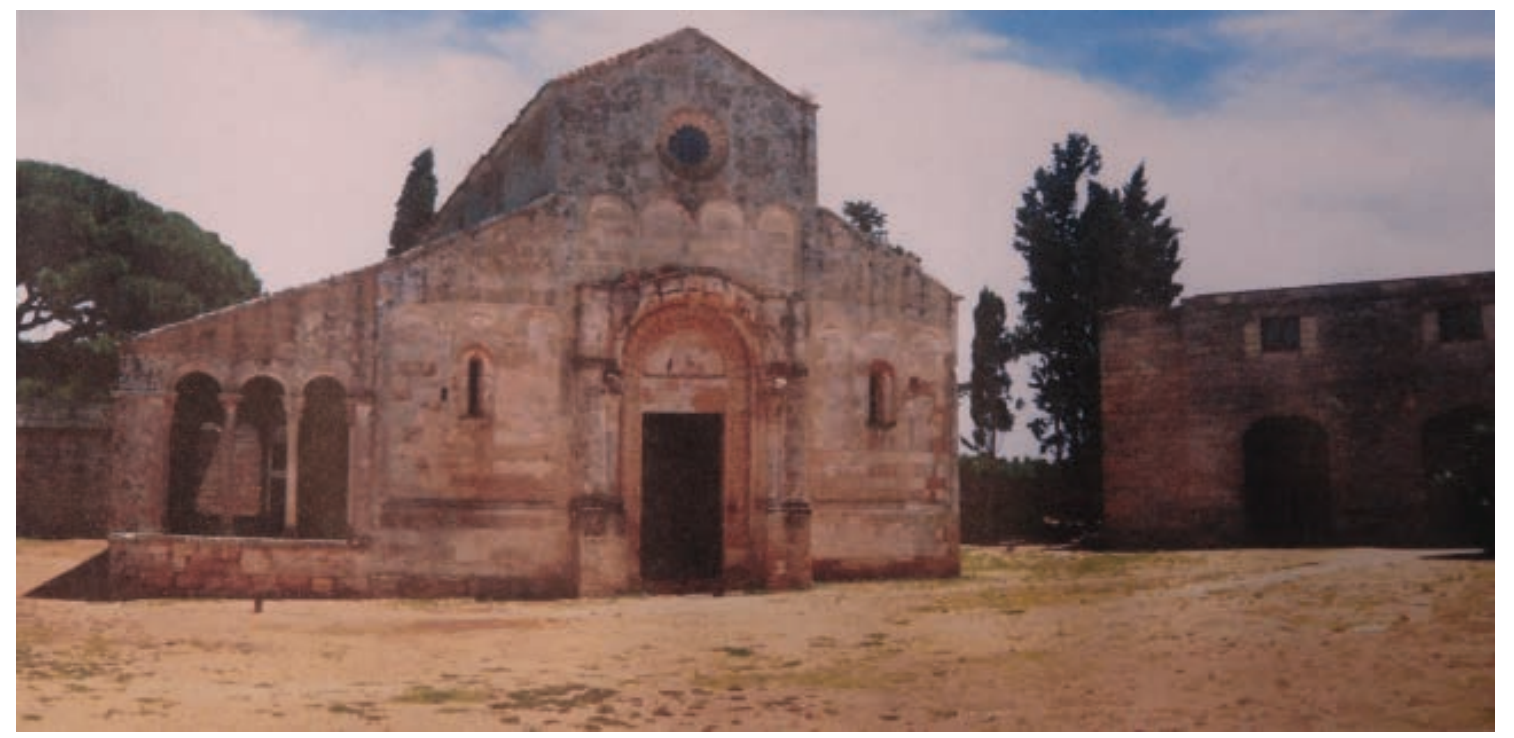

Fig. 5. Squinzano (LE): Chiesa e monastero di Santa Maria delle Cerrate.

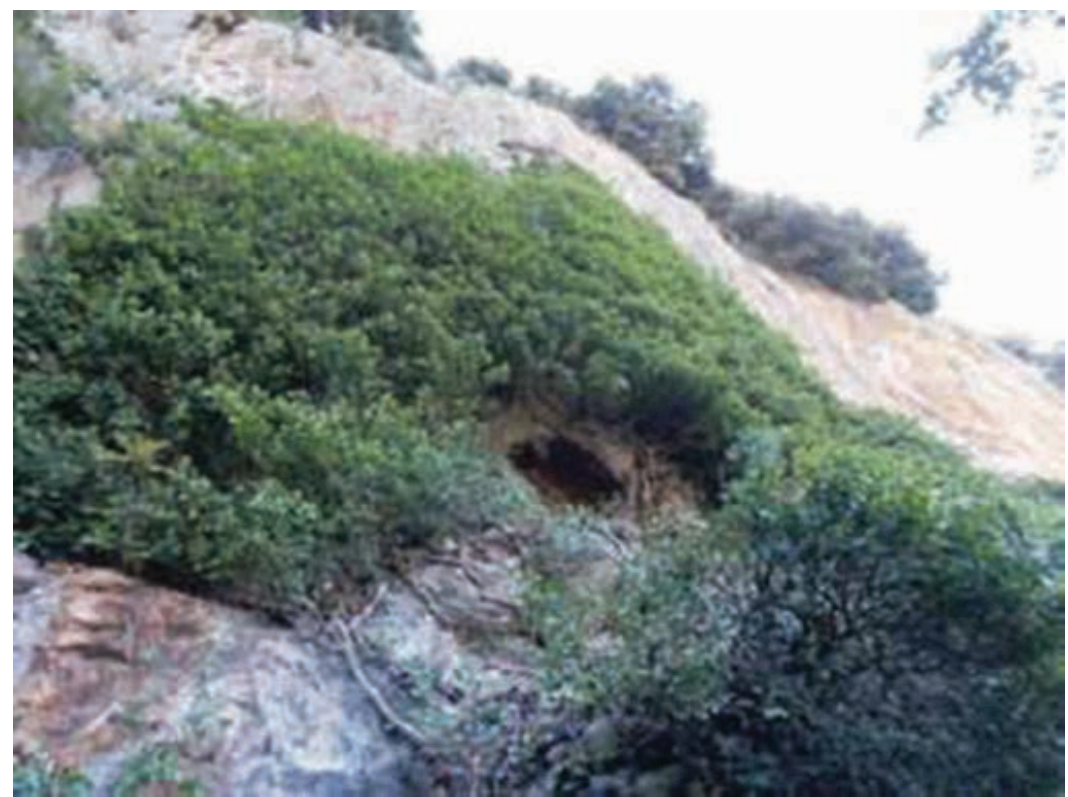

Fig. 6. Lagonegro (PZ): Eremo di San Saba.

compagno il nipote ${ }^{49}$; Luca di Demenna fu raggiunto dalla sorella Caterina, rimasta vedova, e dai suoi figli ${ }^{\circ}$.

Ma il terrore delle incursioni agarene spingeva le popolazioni verso le alture e a fortificare gli abitati. Nel Mercurio, con il suo centro nell'alta valle del Lao, nel Latiniano, con baricentro nel medio corso del Sinni, e nelle valli dell'Agri, del Basento e del Bradano tra X e XI secolo si assiste ad un complesso processo di appropriazione dello spazio da parte dei monaci italo-greci, che, con la loro attività manuale, tra Calvera, Cersosimo, Guardia Perticara, Senise, Teana, Viggiano, Episcopia e Rotondella, costruirono e avviarono ben venti monasteri dotati di terre coltivabili, mulini, frantoi, palmenti e strumenti per l'esercizio di alcuni mestieri fondamentali nell'economia della comunità monastica e del villaggio (maniscalchi, sellai, fabbri ecc.) ${ }^{5}$. Ed è ben documentata anche l'attività aggregante in contesti spopolati

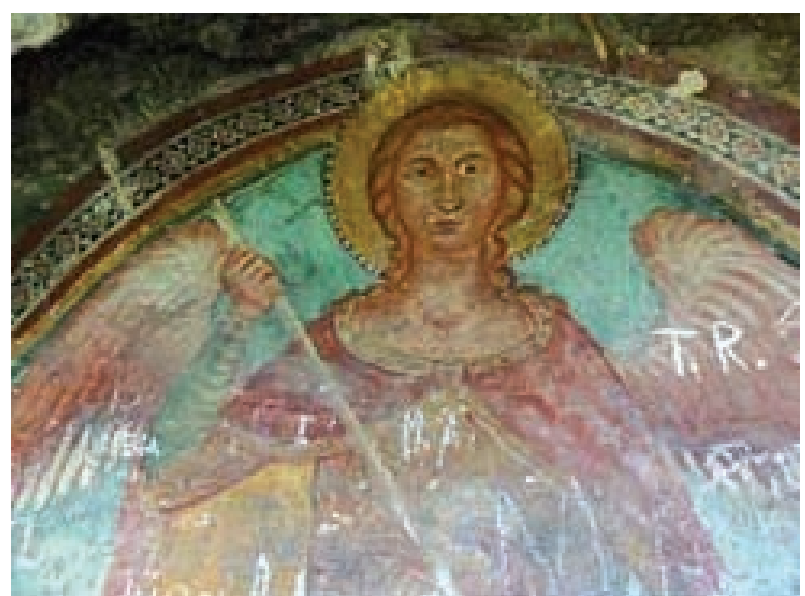

Fig. 7. Lagonegro (PZ): Eremo di San Saba, arcangelo Michele.

o poco abitati dei monaci di Sant'Elia e Anastasio a Carbone, di Sant'Angelo a San Chirico Raparo, di Santa Maria a Kyr-Zosimo, di San Luca ad Armento, di Santa Maria o della Theotokos del Rifugio a Tricarico, di San Luca a Missanello.

Nel Tipikon di San Salvatore di Messina, redatto dall'abate Luca nel 1149, si ricorda che al di fuori del monastero i monaci avevano «costruito un ospedale, uno xenodochio (albergo per i pellegrini) e anche delle arcontaricia (locande), alcuni serbatoi di grano, una panetteria, una cucina, un mulino e alcuni locali per i lavoratori laici»; e poi «piantato olivi, viti, orti, e costruito grandissimi caseggiati nei campi per il raccolto dei prodotti stagionali e abitazioni per i residenti e in alcuni luoghi sanctuaria (chiese rurali) $\aleph^{52}$.

Chiese rurali, s'intende laddove possibile, anche in insediamenti rupestri, sia sub divo che in grotta, a scandire la geografia dei luoghi, le abitudini e le tradizioni locali. E in quelle chiese realizzarono pitture parietali perché servissero ad educare i fedeli alle funzioni liturgiche, come ricordano Nilo di Ancira nel 430 e Gregorio Magno nel 59953. Del resto, la copiatura dei manoscritti era considerata per il monaco un

\footnotetext{
${ }_{49}$ Acta Sanctorum, Martii, II, Bruxellis, 1668, p. 32.

$5^{50}$ Acta Sanctorum, Octobris, IV, Bruxellis, 178o, p. 341.

${ }^{51}$ G. Lunardi, H. Houben, G. Spinelli (a cura di), Monasticon Italiae, III. Puglia e Basilicata, Cesena, 1986, p. 158-203.

${ }^{52}$ GUILLOU, Aspetti della civiltà bizantina op. cit., p. 479.

${ }_{53}$ Nilo di Ancira verso il 430 scrive all'eparco Olimpiodoro: «Tu ti proponi di decorare la chiesa (che hai di recente costruito) con scene di caccia e di pesca per il piacere degli occhi... idea puerile che disorienterà l'occhio dei fedeli; al contrario, tu devi coprire la chiesa di scene dell'Antico Testamento, e, per
} 


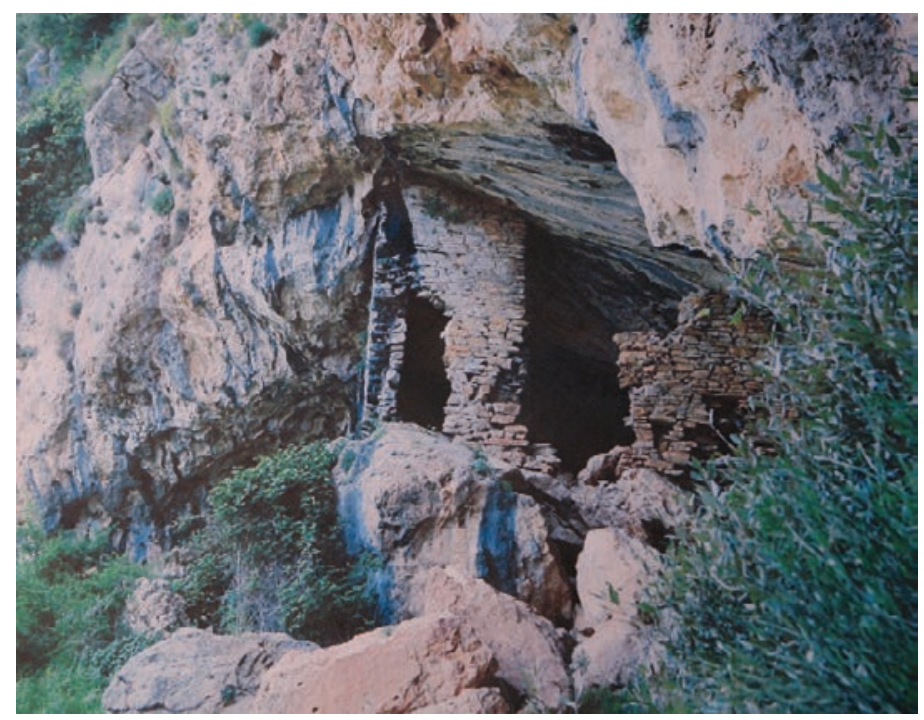

lavoro allo stesso titolo dei lavori manuali che costituivano una parte del programma quotidiano fissato dal Tipikòn di ogni monastero ${ }^{54}$

Quando, poi, dalla fine dell'XI secolo il monachesimo latino via via ridusse gli spazi vitali al monachesimo italo-greco, sino a soffocarlo, nel Mezzogiorno il lavoro manuale divenne sempre più prerogativa dei laici su cui i monaci esercitavano un controllo severo e, spesso, poco caritatevole 55 . La feudalizzazione di numerose abbazie cambiò i connotati delle regole monastiche e le abitudini dei monaci sempre meno avvezzi alle fatiche dei lavori manuali, sempre meno impegnati nei lavori usuranti. E solo con la nascita dell'ordine cistercense (secolo XII), il lavoro manuale acquisì nuova centralità nel sistema aziendale e un posto di rilievo nello spirito del nuovo monachesimo benedettino ${ }^{56}$.

Fig. 8. Orsomarso (CS): Grotta di San Michele, eremo di San Nilo.

questo, rivolgerti al miglior pittore, affinché gli analfabeti che non possono leggere le Sacre Scritture, vedendo le pitture apprendano la forza dei veri servitori di Dio» (Patrologia graeca, 79, col. 577). E Gregorio Magno in una lettera al vescovo di Marsiglia, Sereno, ricorda che la pittura «è adoperata nelle chiese perché gli analfabeti, almeno guardando sulle pareti, leggano ciò che non sono capaci di decifrare sui codici» [GREGORIO MAGNO, Registrum epistularum, D. Norberg (ed.), Turnholti, 1982 (Corpus christianorum. Series latina, CXL A), IX, 209, p. 768].

${ }^{54}$ GUILLOU, Aspetti della civiltà bizantina, op. cit. (n. 36), p. 377.

55 DALENA, Da Matera a Casalrotto, op. cit. (n. 34), p. 109-124.

${ }_{56}^{6}$ P. DALENA, Basilicata cistercense (il codice Barb. Lat. 3247), Galatina 1995 (Itinerari di ricerca storica. Supplementi, 14]; R. COMBA, Le scelte economiche dei monaci bianchi nel Regno di Sicilia (XII-XIII secolo): un modello cistercense?, in H. Houben e B. Vetere (a cura di), I Cistercensi nel Mezzogiorno medievale, Atti del Convegno internazionale di studio in occasione del IX centenario della nascita di Bernardo di Clairvaux (Martano-Latiano-Lecce, 25-27 febbraio 1991), Galatina, 1994, p. 117-164; B. VETERE, Elementi tradizionali e aspetti nuovi nella spiritualità monastica meridionale all'epoca di S. Bernardo, Ibidem, p. 33-72.

\begin{tabular}{|l|l|l|l|l|}
\hline Hortus Artium Mediev. & Vol. 23/1 & $358-365$ & P. Dalena & IL LAVORO MANUALE... \\
\hline
\end{tabular}

